\title{
Uniform Asymptotic Expansions of Confluent Hypergeometric Functions
}

\author{
N. M. Temme \\ Department of Applied Mathematics, Mathematical Centre, \\ 2de Boerhaavestraat 49, Amsterdam. The Netherlands
}

[Received 14 February 1977 and in revised form 4 January 1978]

\begin{abstract}
Asymptotic expansions are derived for the confluent hypergeometric functions $M(a, b, x)$ and $U(a, b, x)$ for large $b$. The results are uniformly valid with respect to $\lambda=x / b$ in a neighbourhood containing $\lambda=1 ; a$ is a fixed parameter. The expansions are derived from integral representations and contain parabolic cylinder functions and asymptotic series.
\end{abstract}

\section{Introduction}

IN A RECENT paper (Temme, 1975) new asymptotic expansions for the incomplete gamma functions $\gamma(\alpha, x)$ and $\Gamma(\alpha, x)$ were derived. The methods immediately lead to results for the confluent hypergeometric functions, of which the incomplete gamma functions are special cases. In the notation of Abramowitz \& Stegun (1964) we denote the confluent hypergeometric functions by $M(a, b, x)$ and $U(a, b, c)$. They are solutions of Kummer's equation

$$
x y^{\prime \prime}+(b-x) y^{\prime}-a y=0 .
$$

We suppose that the parameters are real. Much information on $M$ and $U$ is contained in, for instance, Slater (1960).

For $b \rightarrow \infty$ and $x \rightarrow \infty$ the asymptotic behaviour of solutions of (1.1) depends on the value of $\lambda=x / b$. The critical value is $\lambda=1$. The author's results for the functions $\gamma(\alpha, x)$ and $\Gamma(\alpha, x)$ describe the non-uniform behaviour of these functions adequately, especially near and in $x=\alpha$. In the present paper expansions for $M$ and $U$ are given, which are uniformly valid in a $\lambda$-interval containing $\lambda=1$.

From the literature we know already that asymptotic expansions for $M$ and $U$ (for $b \rightarrow \infty, x \rightarrow \infty, x \sim b$ ) involve parabolic cylinder functions, which are special cases of the confluent hypergeometric functions. Explicitly, we have

$$
D_{v}(x)=2^{v / 2} e^{-x^{2} / 4} U\left(-\frac{1}{2} v, \frac{1}{2}, \frac{1}{2} x^{2}\right) .
$$



The Whittaker functions are closely connected with the confluent hypergeometric
functions. The relations are

$$
\begin{aligned}
& M_{\kappa, \mu}(x)=e^{-x / 2} x^{\mu+\frac{1}{2}} M\left(\frac{1}{2}+\mu-\kappa, 1+2 \mu, x\right) \\
& W_{\kappa, \mu}(x)=e^{-x / 2} x^{\mu+\frac{1}{2}} U\left(\frac{1}{2}+\mu-\kappa, 1+2 \mu, x\right) .
\end{aligned}
$$

Wong (1973) gives an asymptotic expansion of the Whittaker function $W_{\kappa, \mu}(z)$ for large values of the three parameters. In that expansion, parabolic cylinder functions
also occur. For

the expansion is

$$
z \rightarrow \infty, \quad|\arg z|<\pi-\delta, \quad \kappa=o(z), \quad \mu=o\left(z^{\frac{1}{2}}\right)
$$

$$
W_{\kappa, \mu}\left(\frac{1}{2} z^{2}\right) \sim 2^{-\kappa} z^{\frac{1}{2}} \sum_{n=0}^{\infty} \frac{\Gamma\left(\frac{1}{2}+2 \mu+n\right)}{n ! \Gamma\left(\frac{1}{2}+2 \mu-n\right)} \frac{D_{2 \kappa-n-\frac{1}{2}}(z)}{(2 z)^{n}} .
$$

In our expansions we can take both $\kappa$ and $\mu$ of order $O\left(z^{2}\right)$, but we have the condition $|\mu-k| \leqslant M$ for some positive constant $M$. Wong's expansion is easy to handle in a numerical sense, but it does not give an approximation near $\lambda=1$. For this aspect we mention the papers of Kazarinoff (1957), Jorna (1965) and the book of Dingle (1973). The latter gives interesting results (from a practical point of view) for $x<b, x>b$ and also near $x=b$.

Kazarinoff investigated the Whittaker functions for complex variables and for large $|\kappa|,|\mu|$, and $x$ unrestricted, under the hypothesis that $\left(\mu^{2}-\kappa^{2}\right) / \kappa$ be bounded. His rigorous analysis is based upon the methods of $\mathrm{R}$. E. Langer for differential equations. His method is quite different from ours, while his expansions contain first order approximations only. But our approximations are of the same kind, with essentially the same argument in the parabolic cylinder functions. Some of the above-mentioned results of Dingle are based on the work of Jorna
(1965), who also used the differential equations as starting point. Jorna's treatment is rather formal and his results are for the $U$-functions only. The terms of the series in his asymptotic expansion (4.6), however, constitute asymptotic sequences for $b \rightarrow \infty$ under the restriction $x / b-1=o(1)$ (for $b \rightarrow \infty$ ). Hence, in Jorna's results, the domain of uniformity with respect to $\lambda=x / b$ shrinks to the point $\lambda=1$ as $b \rightarrow \infty$.

The aim of the present paper is to enlarge the domain of uniformity with respect to $\lambda$ to compact intervals of $(0, \infty)$ and to give the complete asymptotic expansions for both $U$ and $M$. Another point of interest is that the starting point for the investigations is an integral. Standard integrals for the $U$ - and $M$-functions are not flexible enough for our analysis. Therefore we pay attention to other integral representations in Section 2. In Section 3 we give the saddle point analysis leading to
expansions as in Bleistein (1966).

\section{Contour Integrals}

In this section, we consider integrals of the type

$$
\frac{1}{2 \pi i} \int_{L} e^{s} s^{c}(s-x)^{-a} d s
$$


where $a, c$ and $x$ are real numbers. Throughout this paper we take $x \geqslant 0$ and

$$
c=a-b ;
$$

$a$ and $b$ are the parameters of the confluent hypergeometric functions. $L$ is a contour either so that it is a closed circuit such that the integrand of (2.1) returns to its initial value after $s$ has described the circuit, or so that the integrand vanishes at each limit. Of course, the integral is supposed to converge on L. From well-known methods (cf. Hochstadt, 1971, p. 100) it follows, denoting the integral in (2.1) by $y(x)$, that $y(x)$ satisfies

$$
x y^{\prime \prime}+(b-x) y^{\prime}-a y=\frac{-a}{2 \pi i} \int_{L} \frac{d}{d s}\left[e^{s} s^{c+1}(s-x)^{-a-1}\right] d s,
$$

and hence that it satisfies Kummer's equations (1.1).

After a further specification of $L$, we wish to write (2.1) as a $U$-or $M$-function, or as a linear combination of them. When considering known asymptotic behaviour of $U$ and $M$ for $x \rightarrow 0$ or $x \rightarrow \infty$, we arrive at the following representations (the manyvalued functions in the integrand are supposed to be real for positive values of their arguments, the figures show appropriate branch-cuts)

$$
\frac{1}{2 \pi i} \int_{-\infty}^{(x+)} e^{s} s^{c}(s-x)^{-a} d s=\frac{1}{\Gamma(b)} M(a, b, x),
$$

(with contour as in Fig. 1);

$$
\frac{1}{2 \pi i} \int_{-\infty}^{(0+)} e^{s} s^{c}(x-s)^{-a} d s=\frac{1}{\Gamma(-c)} U(a, b, x),
$$

(with contour as in Fig. 2);

$$
\frac{1}{2 \pi i} \int_{L_{\varepsilon}} e^{s} s^{c}(s-x)^{-a} d s=\frac{e^{\varepsilon i \pi c+x}}{\Gamma(a)} U\left(-c, b, e^{-\varepsilon i \pi} x\right) .
$$

In (2.4), $\varepsilon= \pm 1 ; L_{\varepsilon}$ is sketched in Figs 3 and 4 for $\varepsilon=-1, \varepsilon=+1$, respectively.

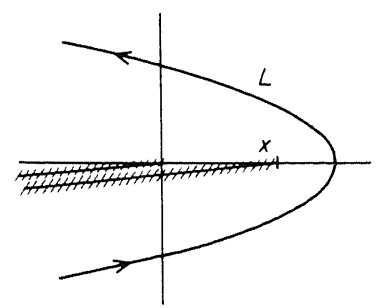

FIG. 1. Contour for (2.2).

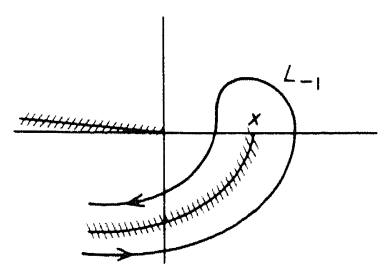

FIG. 3. Contour for $(2.4), \varepsilon=-1$.

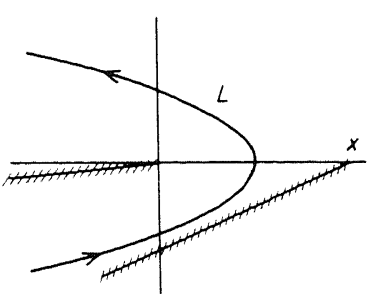

Fig. 2. Contour for (2.3).

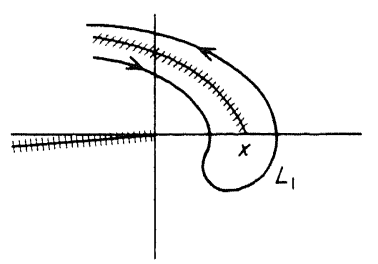

FIG. 4. Contour for (2.4), $\varepsilon=+1$. 


\section{Remarks}

(i) The confluent hypergeometric functions in (2.2), (2.3) and (2.4) are related to each other, as follows from the connection formula

$$
M(a, b, x)=\frac{\Gamma(b)}{\Gamma(b-a)} e^{i \varepsilon \pi a} U(a, b, x)+\frac{\Gamma(b)}{\Gamma(a)} e^{i \pi \varepsilon c+x} U\left(b-a, b, e^{-i \varepsilon \pi} x\right)
$$

where $\varepsilon= \pm 1$. This formula follows from our results by deforming the contour in Fig. 1 into the contours of Figs 2 and 3 (for $\varepsilon=-1$ ) or into those of Figs 2 and 4 (for $\varepsilon=+1)$.

(ii) More integral representations can be derived from (2.1), but in this paper we only use the above ones. Of course, the results (2.2) to (2.4) are valid for wider ranges of the parameters.

The following function is important in the asymptotic expansions considered in this paper:

$$
W_{a}(v)=\int_{-\infty}^{\infty} e^{-\frac{1}{2} u^{2}}(u-v)^{-a} d u,
$$

with $a \in \mathbb{R}, v \in \mathbb{C}$. The contour of integration passes the singularity at $u=v$ as in the following Fig. 5.

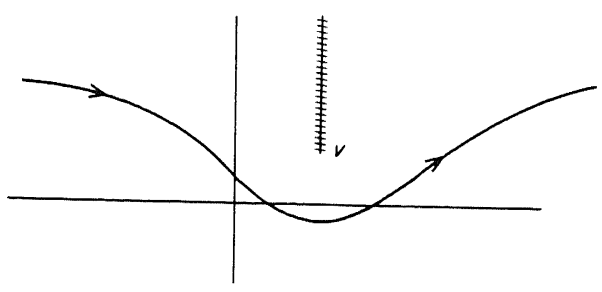

FIG. 5. Contour for (2.6).

As follows from Abramowitz \& Stegun (1964, p. 688), $W_{a}(v)$ is related to the parabolic cylinder function (1.2), this relation being given by

Clearly we have

$$
W_{a}(v)=(2 \pi)^{\frac{1}{2}} \exp \left(-\frac{1}{4} v^{2}+\frac{1}{2} i a \pi\right) D_{-a}(-i v) .
$$

$$
\frac{d}{d v} W_{a}(v)=a W_{a+1}(v)
$$

\section{Uniform Expansions}

\subsection{Saddle Point Contours}

Let us start with $M(a, b, x)$. We will derive an asymptotic expansion of this function for $b \rightarrow \infty$, uniformly valid with respect to $\lambda$, where

$$
\lambda=x / b \text {. }
$$


m (2.2) we obtain

re

$$
M(a, b, x)=\frac{\Gamma(b+1) e^{b} b^{-b}}{2 \pi i} \int_{-\infty}^{\left(\lambda^{+}\right)} e^{b \phi(t)}(1-\lambda / t)^{-a} d t
$$

$$
\phi(t)=t-1-\ln t
$$

us suppose temporarily $\lambda<1$. As in Temme (1975), we choose the contour in ) through the saddle point of $\phi$ at $t=1$ along the steepest descent curve $L$ given $\mathrm{m} \phi(t)=0$, or explicitly

$$
\sigma=\tau \operatorname{cotg} \tau, \quad-\pi<\tau<\pi,
$$

:re $t=\sigma+i \tau(\sigma, \tau \in \mathbb{R})$, see Fig. 6. The function $\phi$ is real and non-positive on $L$.

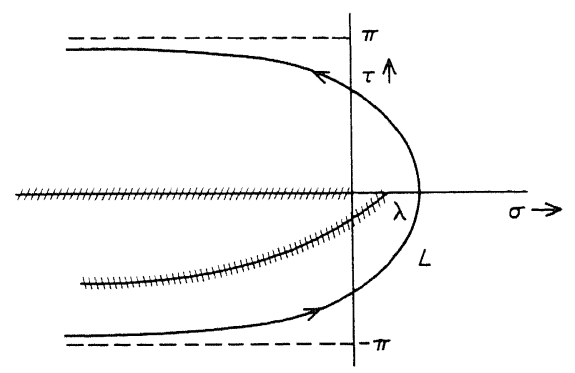

FIG. 6. Contour for (3.2).

t, we define a mapping of the $t$-plane into the $u$-plane by the equation

$$
-\frac{1}{2} u^{2}=\phi(t)
$$

I the condition that $t \in L$ corresponds with $u \in \mathbb{R}$, and $u<0$ if $\tau<0, u>0$ if 0 . From these conditions it follows that

$$
u=i(1-t)\left[2(t-1-\ln t) /(1-t)^{2}\right]^{\frac{1}{2}},
$$

re the square root is positive for positive values of its argument. Integration with ect to $u$ gives for (3.2)

$$
M(a, b, x)=\frac{\Gamma(b+1) e^{b} b^{-b}}{2 \pi i} \int_{-\infty}^{\infty} e^{-\frac{1}{2} b u^{2}} \frac{d t}{d u} \frac{d u}{(1-\lambda / t)^{a}} .
$$

singular points of the integrand in (3.7) are of two different types. First, we have singularity due to the factor $(1-\lambda / t)^{-a}$ (of course, a singularity will only occur of $0,-1,-2, \ldots)$. The singular point $t=\lambda$ in the $t$-plane corresponds with a point $u(\lambda)=u_{1}$, say, in the $u$-plane explicitly given by (cf. (3.6))

$$
u_{1}=i(1-\lambda)\left[2(\lambda-1-\ln \lambda) /(1-\lambda)^{2}\right]^{\frac{1}{2}} \text {, }
$$

if $\lambda \rightarrow 1$, then $u_{1} \rightarrow 0$. The contour in (3.7) is as in Fig. 5, with $v=u_{1}$. If $u_{1}>0$, an ideal contour of integration is the steepest descent path $\operatorname{Im} u=0$. If 
$\operatorname{Im} u_{1} \leqslant 0$, the contour in (3.7) will be deformed around the branch-cut of $(1-\lambda / t)^{-a}$. Hence, we may dispose of the condition $0 \leqslant \lambda<1$ and we suppose henceforth $\lambda \geqslant 0$. The second type of singularities of the integrand is due to the factor $d t / d u$, which,
by using (3.3) and (3.5) can be written as

$$
\frac{d t}{d u}=\frac{u t}{1-t}
$$

The point $t=1$, corresponding to $u=0$, gives a regular point. On account of the many-valuedness of the logarithm in (3.3), we also must consider the points $\exp (2 \pi i n)$, for integer values of $n$, giving a sequence of singular points

$$
2(\pi i n)^{\frac{1}{2}}, \quad n= \pm 1, \pm 2, \ldots,
$$

in the $u$-plane. A proper interpretation of the square root gives that for $n= \pm 1$ the points are $2 \sqrt{\pi} e^{ \pm 3 \pi i / 4}$. When distorting the contour in (3.7) in order to allow nonpositive values of $\operatorname{Im} u_{1}$, the singularities (3.10) must be avoided.

It is important to note that the singularities of the second type, given in (3.10), are fixed points in the $u$-plane, whereas $u_{1}$ given in (3.8) may be close to the origin (the saddle point) $; u_{1}=0$ corresponds with $\lambda=1$. The point $u_{1}$ causes a non-uniform behaviour in (3.7) while the points in (3.10) are of a secondary interest.

\subsection{The Expansion for $M$}

We use an integration by parts procedure suggested by Bleistein (1966). The integral in (3.7) is written as

$$
J\left(a, b, u_{1}\right)=\int_{-\infty}^{\infty} e^{-\frac{1}{2} b u^{2}} G(u) \frac{d u}{\left(u-u_{1}\right)^{a}},
$$

where the contour is as in figure (2.5) (with $v=u_{1}$ ) and

$$
G(u)=\left[\left(u-u_{1}\right) /(1-\lambda / t)\right]^{a} \frac{d t}{d u} .
$$

Except for the points given in (3.10), $G$ is a holomorphic function of $u$. Especially, it is regular at $u=u_{1}$. Straightforward application of Bleistein's method gives the

$$
\begin{aligned}
M(a, b, x) \sim \frac{\Gamma(b+1) e^{b} b^{-b+\frac{1}{2}(a-1)}}{2 \pi i} \times & {\left[W_{a}\left(u_{1} b^{\frac{1}{2}}\right) \sum_{n=0}^{\infty} \gamma_{2 n} b^{-n}+b^{-\frac{1}{2}} W_{a-1}\left(u_{1} b^{\frac{1}{2}}\right) \sum_{n=0}^{\infty} \gamma_{2 n+1} b^{-n}\right] . }
\end{aligned}
$$

$W_{a}(v)$ is given in (2.6). The coefficient $\gamma_{i}$ are functions of $a$ and $\lambda$. The first few are

$$
\begin{aligned}
& \gamma_{0}=\lambda\left[(1-\lambda) / u_{1}\right]^{a-1}, \\
& \gamma_{1}=\left\{\lambda\left[(1-\lambda) / u_{1}\right]^{a-1}-i\left[u_{1} /(\lambda-1)\right]^{a}\right\} / u_{1} .
\end{aligned}
$$


In general, we have for $n>0$

$$
\begin{aligned}
\gamma_{2 n} & =(1-a) G_{n}\left(u_{1}\right), \\
\gamma_{2 n+1} & =(1-a)\left[G_{n}\left(u_{1}\right)-G_{n}(0)\right] / u_{1}+G_{n}^{\prime}(0) .
\end{aligned}
$$

The functions $G_{n}$ are determined recursively from the equations

$$
(1-a) G_{n}(u)+\left(u-u_{1}\right) G_{n}^{\prime}(u)=\gamma_{2 n}+\left(u-u_{1}\right) \gamma_{2 n+1}+u\left(u-u_{1}\right) G_{n+1}(u),
$$

$n=0,1, \ldots$, with $G_{0}(u)=G(u)$ given in (3.12). By inspection, $\gamma_{k}=O(1)$ in $\lambda$ if $\lambda \rightarrow 1$.

By using the more familiar parabolic cylinder functions (1.2) we obtain, by considering (2.7), the asymptotic expansion in which all variables are real

$M(a, b, x) \sim(2 \pi)^{-\frac{1}{2}} \Gamma(b+1) b^{-b+\frac{1}{2}(a-1)} e^{b+\frac{1}{4} h_{5}^{2}} \times$

$$
\left[D_{-a}\left(\zeta b^{\frac{1}{2}}\right) \sum_{n=0}^{\infty} \delta_{2 n} b^{-n}+b^{-\frac{1}{2}} D_{1-a}\left(\zeta b^{\frac{1}{2}}\right) \sum_{n=0}^{\infty} \delta_{2 n+1} b^{-n}\right],
$$

where

$$
\zeta=[2(\lambda-1-\ln \lambda)]^{\frac{1}{2}}(\text { with } \operatorname{sign}(\zeta)=\operatorname{sign}(\lambda-1)), \quad \lambda=x / b
$$

and

$$
\delta_{2 n}=e^{\frac{1}{2} i \pi(a-1)} \gamma_{2 n}, \quad \delta_{2 n+1}=-e^{\frac{1}{2} i \pi a_{2 n+1}}
$$

The first coefficients are

$$
\delta_{0}=\lambda[(1-\lambda) / \zeta]^{a-1}, \quad \delta_{1}=\left\{\lambda[(1-\lambda) / \zeta]^{a-1}-[\zeta /(1-\lambda)]^{a}\right\} / \zeta .
$$

The coefficients $\delta_{i}$ (and $\gamma_{i}$ ) are functions of $a$ and $\zeta$, or of $a$ and $\lambda$. That is, the large parameter $b$ does not occur explicitly in $\delta_{i}$. Following Olver (1975), we call $b$ the asymptotic variable and $a$ and $\zeta$ the non-asymptotic variables, and we consider uniformity of the expansions (3.16) with respect to the non-asymptotic variables. From their construction it follows that $\delta_{i}$ are analytic functions of $a$ and $\zeta$ for all real values of these variables (and also for some complex domains, but that is not considered here). Thus it follows that for $a$ and $\zeta$ belonging to compact sets of the real line the sequences $\left\{\delta_{2 n} b^{-n}\right\}$ and $\left\{\delta_{2 n+1} b^{-n}\right\}$ appearing in (3.16) are uniform asymptotic sequences. From Bleistein (1966; see also Bleistein \& Handelsman, 1975, Chapter 9), the asymptotic nature of the expansion can be examined, giving error bounds and uniformity again on compact sets for real $a$ and $\zeta$.

Translating it in terms of $\lambda$, it says that we have uniformity in compact $\lambda$-intervals of $(0, \infty)$. This extends the results of Jorna (1965), whose expansion admits $\lambda$-values from an interval containing $\lambda=1$, which for $b \rightarrow \infty$ coincides with $\lambda=1$.

\section{Remark}

For $a=1$ the confluent hypergeometric functions are incomplete gamma functions. Explicitly, we have

$$
\gamma(\alpha, x)=\alpha^{-1} x^{\alpha} e^{-x} M(1, \alpha+1, x), \quad \Gamma(\alpha, x)=x^{\alpha} e^{-x} U(1, \alpha+1, x) .
$$

For this case

$$
\begin{aligned}
D_{0}(z) & =e^{-\frac{1}{4} z^{2}}, & D_{-1}(z) & =(\pi / 2)^{\frac{1}{2}} e^{\frac{1}{4} z^{2}} \operatorname{erfc}(z / \sqrt{2}), \\
\delta_{0} & =\lambda, & \delta_{2 n} & =0 \quad(n>0) .
\end{aligned}
$$


As can be verified, expansion (3.17) is the analogue of the result of our previous paper. There it is proved that the expansion for $\alpha \rightarrow \infty$ is uniformly valid in $x \geqslant 0$. Hence, the range of uniformity is larger than in the present paper.

\subsection{The Expansion for $U$}

The starting point is (2.3). After some transformations, we obtain

$$
U(a, b, x)=\frac{b^{1-b} e^{b} \Gamma(b-a)}{2 \pi i} \int_{-\infty}^{\infty} e^{-\frac{1}{2} b u^{2}} G(u) \frac{d u}{\left(u_{1}-u\right)^{a}},
$$
where $G$ and $u_{1}$ are given in (3.12) and (3.8). The contour passes the singularity at
$u=u_{1}$ as in Fig. 7 .

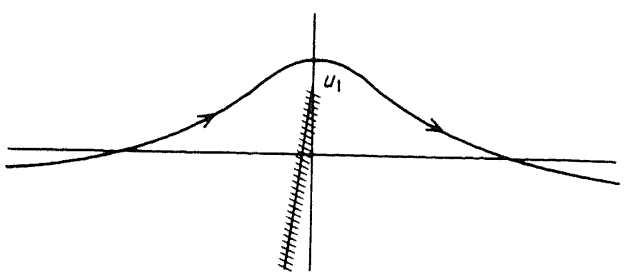

Fig. 7. Contour for (3.18).

The asymptotic expansion now contains functions of the type

$$
\int_{-\infty}^{\infty} e^{-\frac{1}{2} u^{2}}(v-u)^{-a} d u \text {. }
$$

If we make the change of variable $u \rightarrow-u$, it appears that this integral equals $W_{a}(-v)$.

Proceeding as before, we arrive at the expansion

$$
\begin{aligned}
U(a, b, x) \sim & (2 \pi)^{-\frac{1}{2}} b^{\frac{1}{2}(1+a)-b} e^{b+\frac{1}{4} b \zeta^{2}} \Gamma(b-a) \times \\
& {\left[D_{-a}\left(-\zeta b^{\frac{1}{2}}\right) \sum_{n=0}^{\infty} \delta_{2 n} b^{-n}-b^{-\frac{1}{2}} D_{1-a}\left(-\zeta b^{\frac{1}{2}}\right) \sum_{n=0}^{\infty} \delta_{2 n+1} b^{-n}\right] . }
\end{aligned}
$$

The coefficients $\delta_{i}$ are the same as those for $M(a, b, x)$ in (3.16).

The expansions for the integrals along the contours in Figs 3 and 4 follow now from (2.5). By using (3.16), (3.19) and a connection formula for the parabolic cylinder with $\varepsilon= \pm 1$, we obtain

$$
D_{v}(z)=e^{-\varepsilon i v \pi} D_{v}(-z)+(2 \pi)^{\frac{1}{2}} \Gamma(-v)^{-1} D_{-v-1}(\varepsilon i z) e^{-\varepsilon i \pi(v+1) / 2},
$$

$$
\begin{aligned}
& U\left(b-a, b, e^{-\varepsilon i \pi} x\right) \sim b^{\frac{1}{2}(a+1)-b} e^{b+\frac{1}{4} b \zeta_{5}^{2}-x+\frac{1}{2} i \varepsilon \pi(2 b-a-1)} \times \\
& {\left[D_{a-1}\left(\varepsilon i \zeta b^{\frac{1}{2}}\right) \sum_{n=0}^{\infty} \delta_{2 n} b^{-n}+b^{-\frac{1}{2}}(a-1) e^{\frac{1}{2} i \pi \pi} D_{a-2}\left(\varepsilon i \zeta b^{\frac{1}{2}}\right) \sum_{n=0}^{\infty} \delta_{2 n+1} b^{-n}\right] \text {. }}
\end{aligned}
$$


The author would like to thank the referee for mentioning the papers of Jorna and Kazarinoff, which were overlooked in the first version of the paper.

\section{REFERENCES}

Abramowitz, M. \& Stegun, I. A. 1964 Handbook of Mathematical Functions with Formulas, Graphs and Mathematical Tables. Washington D.C.: NBS.

Bleistein, N. 1966 Comm. Pure appl. Math. 19, 353.

Bleistein, N. \& Handelsman, R. A. 1975 Asymptotic Expansions of Integrals. Holt, Rinehart and Winston.

Dingle, R. B. 1973 Asymptotic Expansions: Their Derivation and Interpretation. London: Academic Press.

JoRnA, S. 1965 Proc. R. Soc. London A 284, 531.

KaZARINOFF, N. D. 1957 J. Math. Mech. 6, 341.

Olver, F. W. J. 1975 In Theory and Applications of Special Functions (Ed. R. A. Askey). New York: Academic Press.

Slater, L. J. 1960 Confluent Hypergeometric Functions. Cambridge University Press.

Temme, N. M. 1975 Math. Comp. 29, 1109.

Wong, R. 1973 Math. Comp. $27,429$. 